\title{
miR-204 functions as a tumor suppressor gene, at least partly by suppressing CYP27A1 in glioblastoma
}

\author{
JUN XIN $^{1 *}$, LI-MIN ZHENG ${ }^{2 *}$, DE-KE SUN ${ }^{1}$, XIAN-FENG LI ${ }^{1}$, \\ PENG XU ${ }^{1}$ and LI-QIANG TIAN ${ }^{3}$ \\ ${ }^{1}$ Department of Neurosurgery, Yishui Central Hospital, Linyi, Shandong 276400; \\ ${ }^{2}$ Department of Neurosurgery, Tai'an Central Hospital, Tai'an, Shandong 271000; \\ ${ }^{3}$ Department of Neurosurgery, Linyi People's Hospital, Linyi, Shandong 276003, P.R. China
}

Received September 23, 2015; Accepted March 14, 2017

DOI: $10.3892 / \mathrm{ol} .2018 .8846$

\begin{abstract}
Gliomas are the most common type of malignant primary brain tumors in adults and exhibit a spectrum of aberrantly aggressive phenotypes. Despite advances in treatments during past decades, prognosis of the disease remains poor, with a median survival time of 12-14 months. Future studies on the molecular mechanism of the disease may provide the theoretical basis to identify new targets for effective therapies. The present study revealed that in glioblastoma cells, the overexpression of cytochrome P450, family 27, subfamily A, polypeptide 1 (CYP27A1) promoted proliferation, while silencing of CYP27A1 inhibited proliferation, without affecting migration and invasion. CYP27A1 protein was upregulated in glioblastoma tissues, indicating that CYP27A1 is an oncogene. The downregulation of specific microRNAs (miRNA) may contribute to the upregulation of oncogenes in glioblastoma. A common strategy was used to predict target miRNAs of CPY27A1 using the miRanda algorithm. miR-211 and miR-204 could target the 3'untranslated region of CPY27A1 mRNA. Additional studies confirmed that the overexpression of miR-204 inhibited CPY27A1 expression in glioblastoma cells. Finally, it was identified that miR-204 was downregulated in glioblastoma and that its overexpression inhibited proliferation, migration and invasion in glioblastoma cells. Thus, it was concluded that miR-204 functions as a tumor suppressor gene, at least partly by suppressing CYP27A1 in glioblastoma.
\end{abstract}

Correspondence to: Dr Li-Qiang Tian, Department of Neurosurgery, Linyi People's Hospital, 27 Jiefang Road, Lanshan, Linyi, Shandong 276003, P.R. China

E-mail: tianliqiang276@126.com

${ }^{*}$ Contributed equally

Key words: glioblastoma, cytochrome P450, family 27, subfamily A, polypeptide 1, microRNA-204, proliferation

\section{Introduction}

Glioblastoma multiforme is the most common type of malignant primary brain tumor in adults (1). Mean progression-free survival is just over 6 months; treatment with surgical resection, chemotherapy and radiation is invariably followed by tumor recurrence (2). Despite the improvement in outcomes with this combined chemoradiotherapy approach, few patients survive beyond 5 years (3). Therefore, future studies on the molecular mechanism of the disease may provide the theoretical basis to identify new targets for effective therapies.

Cytochrome P450, family 27, subfamily A, polypeptide 1 (CYP27A1), also termed CTX\CP27\CYP27 is a ubiquitously expressed mitochondrial enzyme belonging to the cytochrome P450 family (4). CYP27A1 catalyzes the hydroxylation of cholesterol at C-27 to form 27-hydroxycholesterol and cholestenoic acid (5). A single serum measurement of 27-hydroxycholesterol can reliably estimate average levels over a one-year period (6). The role of CYP27A1 in bile acid synthesis in the liver is well established; it catalyzes the initial, rate-limiting step in the alternative bile acid synthetic pathway and the intermediate step in the classic bile acid synthetic pathway $(7,8)$. CYP27A1 is strongly expressed in macrophages within human benign and malignant mammary tissue (4). Breast cancer cell intrinsic expression of CYP27A1 protein is associated with tumor grade (4). However, its roles remain unclear in glioblastoma.

MicroRNAs (miRNAs/miRs) are a class of small RNA found in a diverse range of eukaryotes, including animals, plants and DNA viruses, which range in size between 19 and 24 nucleotides (nts) (9). Aberrant miRNA expression has been linked to glioblastoma (10). miR-204, a direct negative regulator of ezrin gene expression, inhibits glioma cell migration and invasion (11). However, studies continue to emerge with an improved understanding on the mechanism of miR-204 as a tumor suppressive gene in glioblastoma. In the present study, it was observed that CYP27A1 overexpression promoted proliferation, while silencing of CYP27A1 inhibited proliferation in glioblastoma cells, without affecting migration and invasion. It was revealed that CYP27A1 was upregulated in glioblastoma tissues, indicating that CYP27A1 may be an oncogene. The downregulation of specific miRNAs may contribute to the 
upregulation of oncogenes in glioblastoma (12). A common strategy was used to predict target miRNAs of CPY27A1 using the miRanda algorithm. It was revealed that miR-211 and miR-204 could target the 3'untranslated region (UTR) of CPY27A1 mRNA. Overexpressing miR-204 inhibited CPY27A1 expression in glioblastoma cells. Lastly, the present study demonstrated that miR-204 was downregulated in glioblastoma and its overexpression inhibited proliferation, migration and invasion in glioblastoma cells. Therefore, miR-204 functions as a tumor suppressor gene, at least partly by suppressing CYP27A1 expression in glioblastoma.

\section{Materials and methods}

Glioblastoma tissues, cells, CYP27A1-expressing plasmids and shorthairpin(sh)CYP27A1 plasmids. A total of 7 Chinese women patients with glioblastoma were recruited from the Department of Neurosurgery, Yishui Central Hospital (Shandong, China). The mean age was 56 years (range, 31-78 years). All tissues were examined histologically, and pathologists confirmed the diagnosis. The present study was approved by the medical ethics committee of Yishui Central Hospital (Shandong, China). Written informed consent was obtained from each individual. The human glioblastoma U87MG cell line was purchased from the Cell Bank of the Chinese Academy of Sciences (Beijing, China). CYP27A1-expressing plasmids/pcDNA3.1 (pcDNA3.1) and shCYP27A1/scramble were purchased from Tiangen Biotech Co., Ltd. (Shanghai, China).

Protein extraction and western blot analysis. Following transfection, cells and tissues were lysed for $48 \mathrm{~h}$ using RIPA Lysis and Extraction Buffer (Beijing Solarbio Science \& Technology Co., Ltd., Beijing, China), and the protein concentration was measured using Pierce ${ }^{\mathrm{TM}}$ BCA Protein Assay kit (Thermo Fisher Scientific, Inc.). Following heating at $100^{\circ} \mathrm{C}$ for $10 \mathrm{~min}$ in the presence of a loading buffer, equal amounts of protein lysates $(50 \mu \mathrm{g})$ were separated using $10 \%$ SDS-PAGE (Bio-West Inc., Logan, UT, USA) at $100 \mathrm{~V}$ for $1 \mathrm{~h}$, and transferred onto Invitrogen nitrocellulose membranes (Thermo Fisher Scientific, Inc.) at $120 \mathrm{~V}$ for $1 \mathrm{~h}$. Following blocking with $5 \%$ skimmed milk (diluted with PBS), the membranes were incubated overnight at $4^{\circ} \mathrm{C}$ with the following primary antibodies: CYP27A1 (ab126785; 1:500; Abcam, Cambridge, MA, USA), c-myc (ab32072; 1:500; Abcam), RB (ab181616; 1:500; Abcam), Ki-67 (ab15580; 1:500; Abcam), CDK2 (ab32147; 1:500; Abcam), p21 (ab109520; 1:500; Abcam), p53 (ab32049; 1:500; Abcam), PDCD4 (ab51495; 1:500; Abcam), SOX2 (ab92494; 1:500; Abcam), $\beta$-actin (ab8227; 1:500; Abcam). Subsequently, the membranes were incubated with secondary goat monoclonal (RMG01) to rabbit IgG Fab region (Biotinylated; ab222772; 1:10,000; Abcam) at room temperature for $2 \mathrm{~h}$, and proteins were detected using enhanced chemiluminescence (Pierce ${ }^{\mathrm{TM}}$ ECL Western Blotting Substrate; Thermo Fisher Scientific, Inc.).

MTT assay. A total of $5 \times 10^{3}$ cells were seeded onto 96-well plates and transfected with CYP27A1 expressing plasmids or empty vector at $37^{\circ} \mathrm{C}$ for $24 \mathrm{~h}$

Subsequent to transfection, MTT (5 mg/ml; Sigma-Aldrich; Merck KGaA, Darmstadt, Germany) was added to the wells
(20 $\mu \mathrm{l} /$ well) and incubated for $4 \mathrm{~h}$ at $37^{\circ} \mathrm{C}$, and then the supernatants were removed and $2 \%$ dimethyl sulfoxide was added to each well. The absorbance of each sample was measured using a UV spectrophotometer at the wavelength of $490 \mathrm{~nm}$.

Bromodeoxyuridine (BrdU) labeling and immunofluorescence. Cells grown on coverslips (Thermo Fisher Scientific, Inc., Waltham, MA, USA) were incubated with BrdU at $37^{\circ} \mathrm{C}$ for $1 \mathrm{~h}$ and stained with anti-BrdU antibody (1:200; cat. no. ab220076; Abcam) according to the manufacturer's protocol. For immunofluorescence analysis, cells were plated on glass coverslips. Following transfection, cells were exposed to anti-CYP27A1 antibody (1:250; ab198970; Abcam) overnight at $4^{\circ} \mathrm{C}$ and goat anti-rabbit secondary antibody (1:500; ab150079; Abcam). Coverslips were counterstained with DAPI (Molecular Probes; Thermo Fisher Scientific, Inc.) to detect cell nuclei. Microscopic analysis was performed with a confocal laser-scanning microscope (Leica Microsystems, Bensheim, Germany). Fluorescence intensities were measured in three randomly selected viewing areas for 200 300 cells/coverslip and analyzed using ImageJ (version $1.37 \mathrm{v}$; National Institutes of Health, Bethesda, MA, USA).

Migration and invasion assay. Cell suspension (total cells, $3 \times 10^{4}$ ) in medium without fetal bovine serum (FBS) was plated into the top chamber with $8-\mu \mathrm{m}$ pore sized filter inserts of Transwell plates (24-well; Costar, Cambridge, MA, USA). For the Transwell migration assay, the top surface of filter membranes was not coated with Matrigel, while the top surface of filter membranes was coated with Matrigel matrix (BD Biosciences, San Jose, CA, USA) in the Matrigel invasion assay. Medium containing 10\% FBS (Sigma-Aldrich; Merck $\mathrm{KGaA}$ ) was added to the bottom chambers in the two assays. The cells were incubated for $24 \mathrm{~h}$ at $37^{\circ} \mathrm{C}$, and then the non-migrated or non-invaded cells on top surface of the membrane were removed with a cotton swab, the cells under the filter membrane were fixed with $4 \%$ paraformaldehyde for $10 \mathrm{~min}$ at room temperature and stained with $0.2 \%$ crystal violet for $10 \mathrm{~min}$ at room temperature (Sigma-Aldrich; Merck $\mathrm{KGaA})$.

Methods of bioinformatics. The analysis of potential microRNA target sites was performed using the commonly used miRanda prediction algorithm (http://www.microrna.org/).

Reverse transcription-polymerase chain reaction (RT-PCR). Extraction of total RNA and detection of the mature form of miRNAs was performed with the mirVanami RNA Isolation kit (Ambion; Thermo Fisher Scientific, Inc.) according to the manufacturer's protocol. Template DNA was obtained from Dr Xin Shao (Department of Medicine, Jiujiang College, Jiujiang, China). Taq DNA polymerase was purchased from Promega Corporation (Madison, WI, USA). $\beta$-actin was used as an experimental control gene. SYBR green (Takara Biotechnology Co., Ltd., Dalian, China) was used as a fluorophore. Quantification was performed using the $2^{-\Delta \Delta C q}$ method (13).

Luciferase (Luc) assays. Total RNA was isolated from the U87MG cells using TRIzol reagent (cat no. 101472; Invitrogen; Thermo Fisher Scientific, Inc.). cDNA was synthesized from 
A

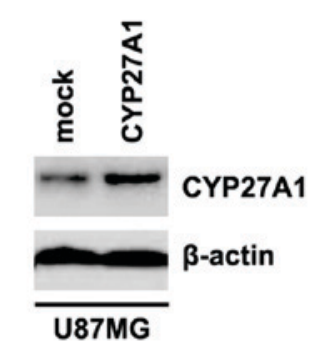

D
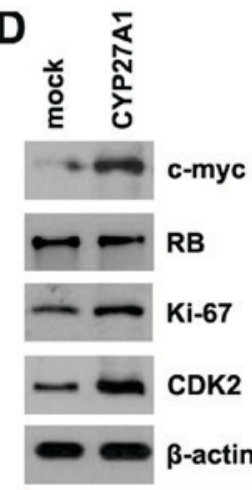

B

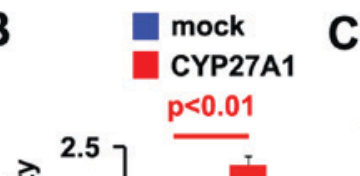

C
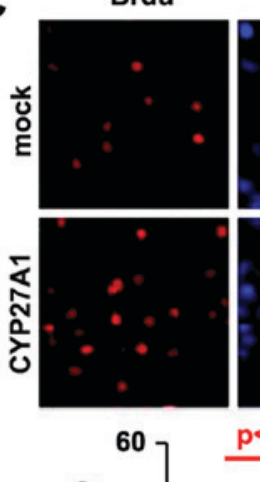

DAPI
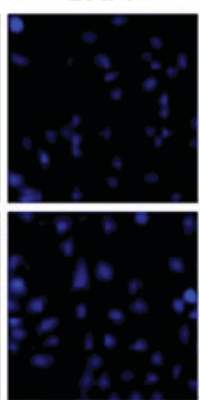

$p<0.01$

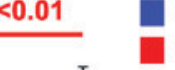

Merge
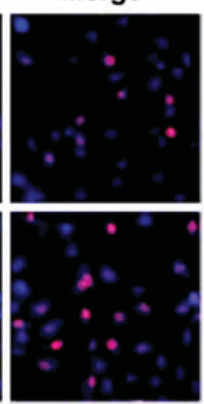

mock

CYP27A1

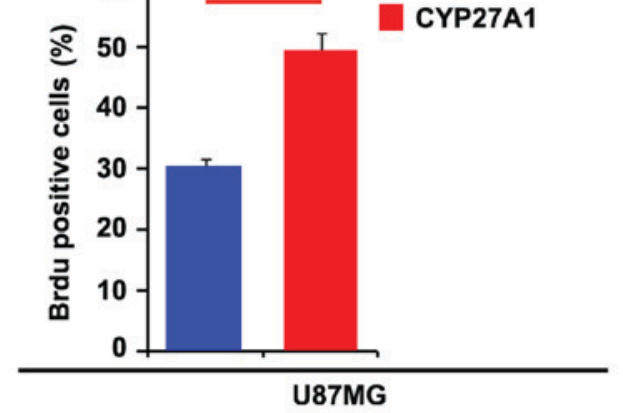

p53

U87MG

PDCD4

Sox2

$\beta$-actin

U87MG

Figure 1. CYP27A1 promotes proliferation in glioblastoma U87MG cells. (A) Western blot analysis for CYP27A1 in U87MG cells. U87MG cells were transfected with CYP27A1-expressing plasmids or the empty vector (mock). $\beta$-actin was used as the loading control. $n=3$. (B) MTT assay for U87MG cells. U87MG cells were transfected with CYP27A1-expressing plasmids or the empty vector (mock) and cellular viability was then measured at the indicated time points using an MTT assay. $\mathrm{n}=3$. (C) BrdU incorporation assay for U87MG cells. Representative micrographs and quantification of BrdU incorporating-cells following transfection with CYP27A1-expressing plasmids or the empty vector (mock). n=3. (D) Western blot analysis for c-myc, RB, Ki67, CDK2, p21, p53, PDCD4 and SOX2 in U87MG cells transfected with CYP27A1-expressing plasmids or the empty vector (mock). $\beta$-actin was used as the loading control. $\mathrm{n}=3$. Error bars indicate standard error of the mean. CYP27A1, cytochrome P450, family 27, subfamily A, polypeptide 1; BrdU, bromodeoxyuridine; RB, retinoblastoma; CDK2, cyclin-dependent kinase 2; PDCD4, programmed cell death protein 4; SOX2, sex determining region Y-box 2.

$1 \mu \mathrm{g}$ of total RNA in a $20 \mu \mathrm{l}$ reverse transcription system followed by PCR amplification in a $50 \mu 1$ PCR system performed using an RT-PCR kit (cat no. A3500; Promega Corporation). The 3'UTR of CYP27A1 was amplified [10X buffer $3 \mathrm{ul}, \mathrm{MgCl}_{2}(25 \mathrm{mM}) 3 \mathrm{ul}$, dNTP $(25 \mathrm{mM}) 0.36 \mathrm{ul}$, forward primer $(10 \mu \mathrm{M}) 1 \mathrm{ul}$, reverse primer $(10 \mu \mathrm{M}) 1 \mu \mathrm{l}$, Taq enzyme (5 U/ $\mu \mathrm{l}) 0.3 \mu \mathrm{l}, \mathrm{ddH}_{2} \mathrm{O} 19.34 \mu \mathrm{l}$ and cDNA $2 \mu \mathrm{l} ; 95^{\circ} \mathrm{C}$ for $2 \mathrm{~min} ; 95^{\circ} \mathrm{C}$ for $30 \mathrm{sec} ; 55^{\circ} \mathrm{C}$ for $60 \mathrm{sec} ; 72^{\circ} \mathrm{C}$ for $\left.30 \mathrm{sec}\right]$ using cDNA from U87MG cells, cloned into pRL-TK (Promega Corporation), checked for orientation, sequenced and termed Luc-CYP27A1-wild-type (WT). For reporter assays, U87MG cells were transiently transfected with WT-reporter plasmid and precursor (pre)-miR-204 using Lipofectamine 2000 (Invitrogen; Thermo Fisher Scientific, Inc.). Reporter assays were performed $36 \mathrm{~h}$ post-transfection using the dual-luciferase assay-system (Promega Corporation), normalized for transfection efficiency by co-transfected Renilla-luciferase.

Northern blot analysis. Northern blot analysis of miRNAs was performed as described previously (14). Probes were labeled with $(\gamma-32 \mathrm{P})$ ATP complementary to miR-204 and U6 small nuclear RNA.
Statistical analysis. All experimental data are presented as the mean \pm standard error, with the number of independent experiments $(n=3)$, and were analyzed using Student's t-test. $P<0.05$ was considered to indicate a statistically significant difference. Analysis was performed using GraphPad Prism software (version 5.0; GraphPad Software, Inc., La Jolla, CA, USA).

\section{Results}

CYP27A1 promotes proliferation of glioblastoma cells. To investigate whether CYP27A1 could promote the proliferation of glioblastoma U87MG cells, firstly using western blot analysis, it was examined whether CYP27A1-expressing plasmids could stably express CYP27A1 protein in U87MG cells. The results demonstrated that CYP27A1 protein could be significantly increased by CYP27A1-expressing plasmids in the cells (Fig. 1A). In order to identify the effect of CYP27A1 on proliferation, an MTT assay was performed. The results demonstrated that overexpression of CYP27A1 significantly upregulated the proliferation rate of U87MG cells following transfection (Fig. 1B). To show the effects of CYP27A1 on proliferation, a BrdU incorporation assay was performed to analyze its effects 
A

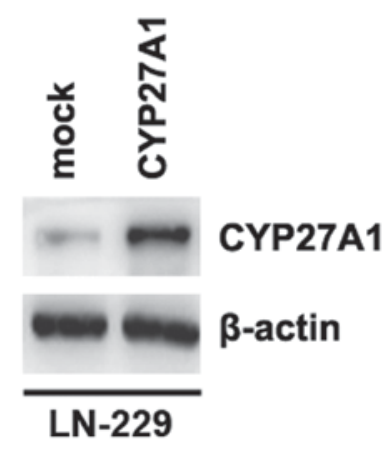

B

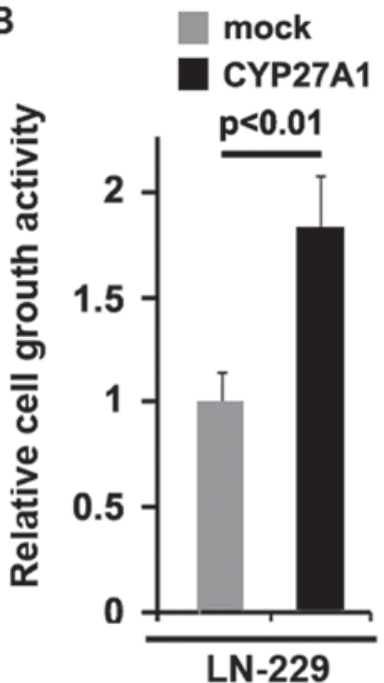

Figure 2. CYP27A1 promotes proliferation in glioblastoma LN-229 cells. (A) Western blot analysis for CYP27A1 in LN-229 cells. LN-229 cells were transfected with CYP27A1-expressing plasmids or the empty vector (mock). $\beta$-actin was used as the loading control. $\mathrm{n}=3$. (B) MTT assay for LN-229 cells. LN-229 cells were transfected with CYP27A1-expressing plasmids or the empty vector (mock) and cellular viability was then measured at the indicated time-points using an MTT assay. n=3. Error bars indicate standard error of the mean.

on DNA synthesis. The results confirmed that CYP27A1 significantly promoted DNA synthesis in the cells, and representative micrographs and quantification of BrdU incorporating-cells following transfection with CYP27A1 or empty vector (mock) are shown (Fig. 1C). Subsequently, western blot analysis was performed to identify whether proliferation-associated markers were affected by CYP27A1 in the cells. The results of western blot analysis revealed that c-myc, sex determining region Y-box 2 (SOX2), Ki-67 and cyclin-dependent kinase 2 were promoted and p21, p53and programmed cell death protein 4 (PDCD4) were inhibited by CYP27A1 (Fig. 1D). Meanwhile, it was observed that CYP27A1 protein was increased by CYP27A1 expression plasmids (Fig. 2A) and overexpression of CYP27A1 promoted proliferation in LN-229 glioblastoma cells (Fig. 2B). These data supported that CYP27A1 promoted proliferation in glioblastoma cells.

CYP27A1 overexpression does not affect migration and invasion in glioblastoma cells. Considering that CYP27A1 evidently promoted U87MG cellular proliferation, it was then determined whether CYP27A1 has an impact on the migration and invasion of glioblastoma cells. The migration and invasion assay results demonstrated that the overexpression of CYP27A1 did not affect migration and invasion in U87MG and LN-229 cells (Fig. 3A and B).

Silencing CYP27A1 inhibits proliferation in glioblastoma cells. It was demonstrated that CYP27A1 overexpression promoted proliferation in U87MG and LN-229 cells. To provide additional evidence that CYP27A1 is involved in the proliferation of glioblastoma cells, the effects of an inhibitor of CYP27A1, shCYP27A1, were studied. Following stable transfection, CYP27A1 expression was detected by western blot analysis. The results demonstrated that exogenous shCYP27A1 significantly downregulated CYP27A1 expression in U87MG cells (Fig. 4A). An MTT assay was performed to detect the proliferation of U87MG cells transfected
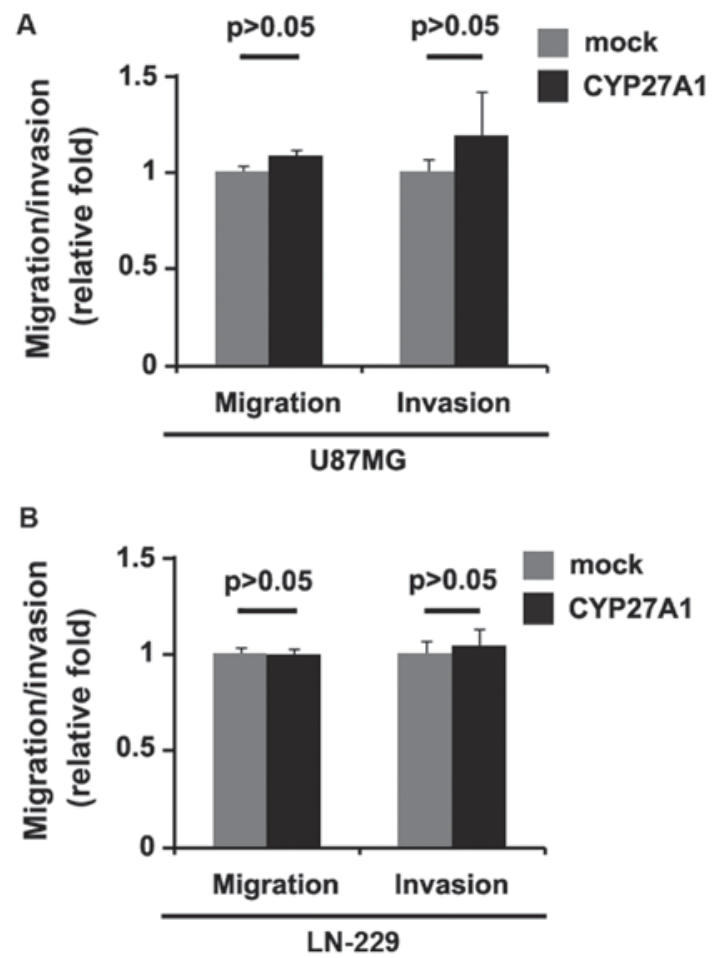

Figure 3. CYP27A1 overexpression does not affect migration and invasion in glioblastoma U87MG and LN-229 cells. (A) Matrigel invasion and Transwell migration assays for U87MG cells transfected with CYP27A1-expressing plasmids or the empty vector (mock). $n=3$. (B) Matrigel invasion and Transwell migration assays for LN-229 cells transfected with CYP27A1-expressing plasmids or the empty vector (mock). $n=3$. Error bars indicate standard error of the mean. CYP27A1, cytochrome P450, family 27, subfamily A, polypeptide 1 .

with shCYP27A1 and scramble. The results demonstrated that shCYP27A1 inhibited proliferation in U87MG cells compared with scramble-transfected groups, and that the inhibition was dose-dependent (Fig. 4B). To demonstrate the effects of silencing CYP27A1 on cellular proliferation, 

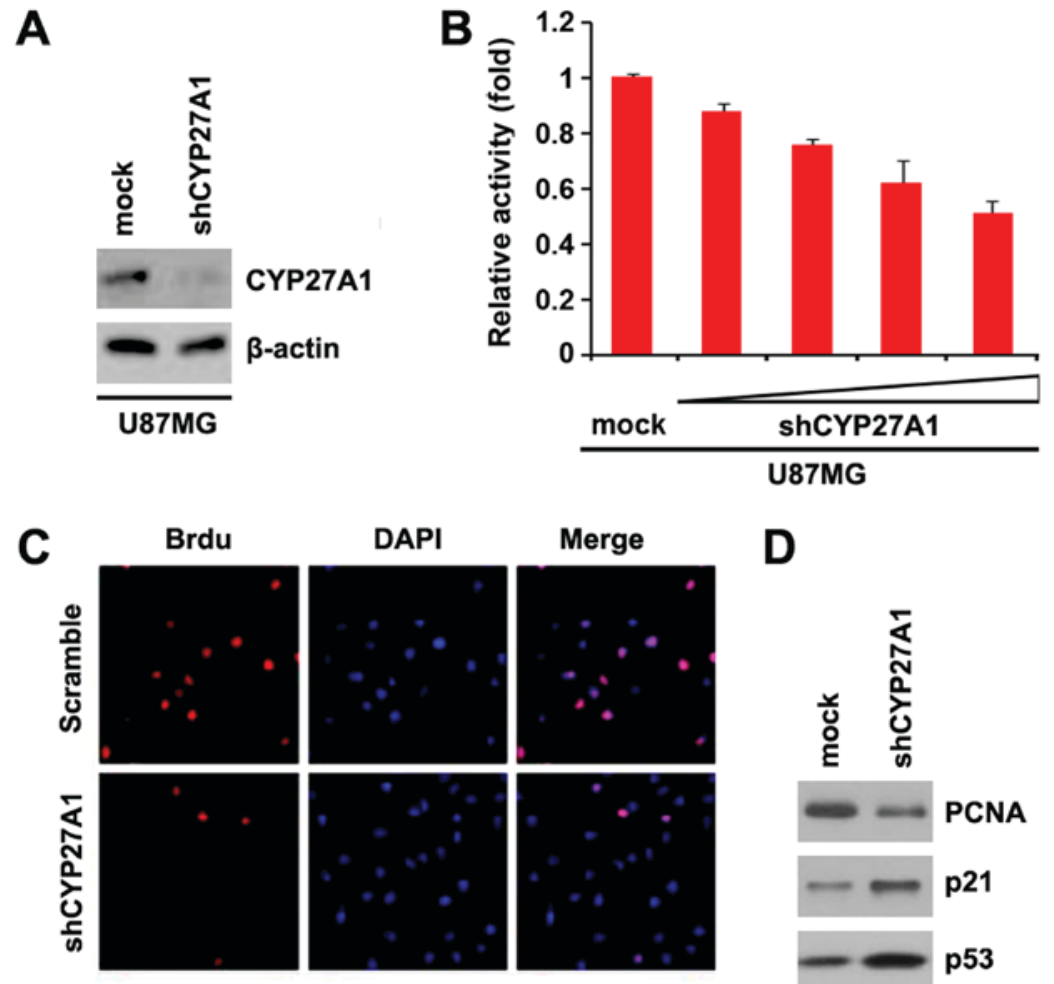

D
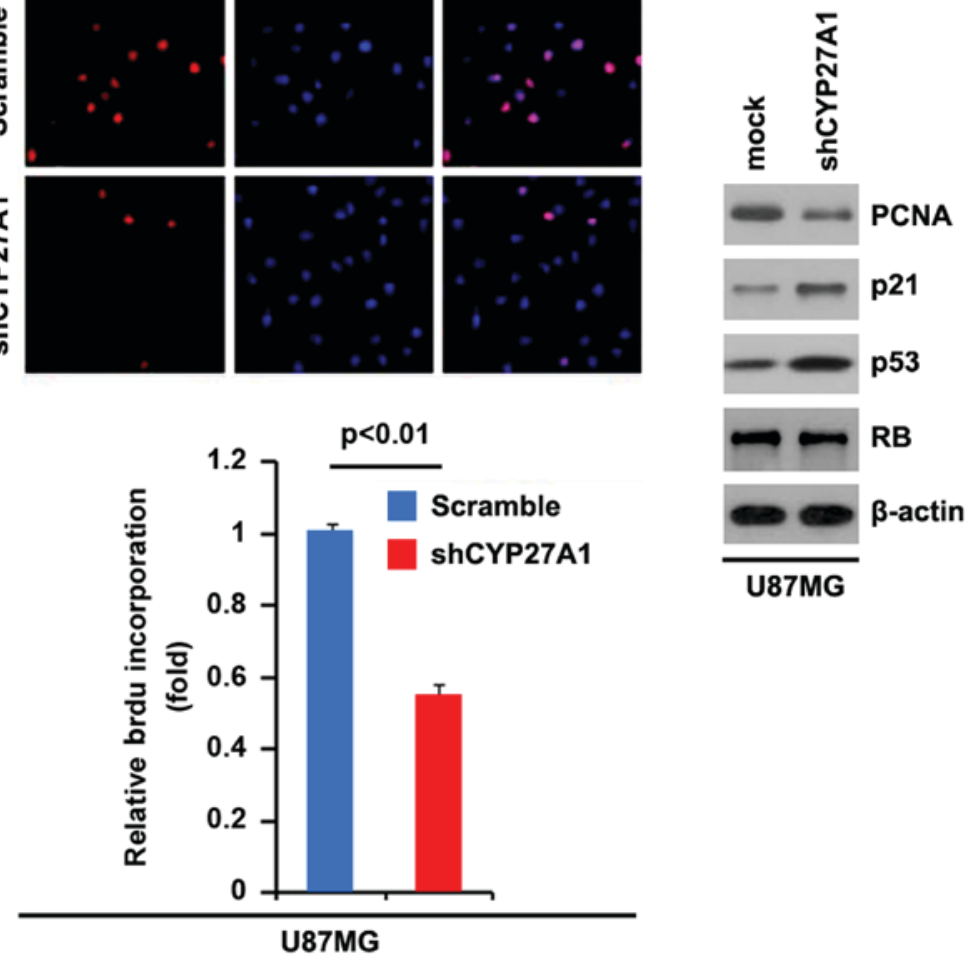

Figure 4. Silencing CYP27A1 inhibits proliferation in glioblastoma U87MG cells. (A) Western blot analysis for CYP27A1 in U87MG cells infected with shCYP27A1 or scramble. $\beta$-actin was used as the loading control. $n=3$. (B) MTT assay for U87MG cells transfected with shCYP27A1 or scramble. $n=3$. (C) BrdUincorporation assay for U87MG cells. Representative micrographs and quantification of BrdU incorporating cells following transfection with shCYP27A1 or scramble. $n=3$. (D) Western blot analysis for PCNA, p21, p53 and RB in U87MG cells transfected with shCYP27A1 or scramble. $\beta$-actin was used as the loading control. $\mathrm{n}=3$. Error bars indicate standard error of the mean. CYP27A1, cytochrome P450, family 27, subfamily A, polypeptide 1; BrdU, bromodeoxyuridine; sh, short hairpin; PCNA, proliferating cell nuclear antigen; RB, retinoblastoma.

BrdU incorporation assay was performed to detect DNA synthesis in the cells. The results confirmed that shCYP27A1 significantly suppressed DNA synthesis in the cells and representative micrographs and quantification of $\mathrm{BrdU}$ incorporating-cells following transfection with shCYP27A1 or scramble are presented (Fig. 4C). Subsequently, western blot analysis was performed to identify whether proliferation-associated markers were affected by shCYP27A1 in the cells. The results of western blot analysis demonstrated that the proliferating cell nuclear antigen was downregulated and p21, as well as p53, were upregulated by silencing CYP27A1 (Fig. 4D).

Silencing CYP27A1 does not affect migration and invasion in glioblastoma cells. It was then determined whether silencing
CYP27A1 would have any impact on migration and invasion in U87MG cells. The migration and invasion assays revealed that silencing CYP27A1 did not affect migration and invasion of them (Fig. 5).

Aberrant expression of CYP27A1 in glioblastoma tissues. In order to identify CYP27A1 protein expression in glioblastoma tissues, western blot analysis was performed to detect CYP27A1 protein between glioblastoma tissues and adjacent normal tissues. It was revealed that CYP27A1 was increased in cancer tissues of 6 patients, compared with adjacent normal tissues (Fig. 6).

CYP27A1 is a target of miR-204 in glioblastoma U87MG cells. Having demonstrated that CYP27A1 expression is specifically 


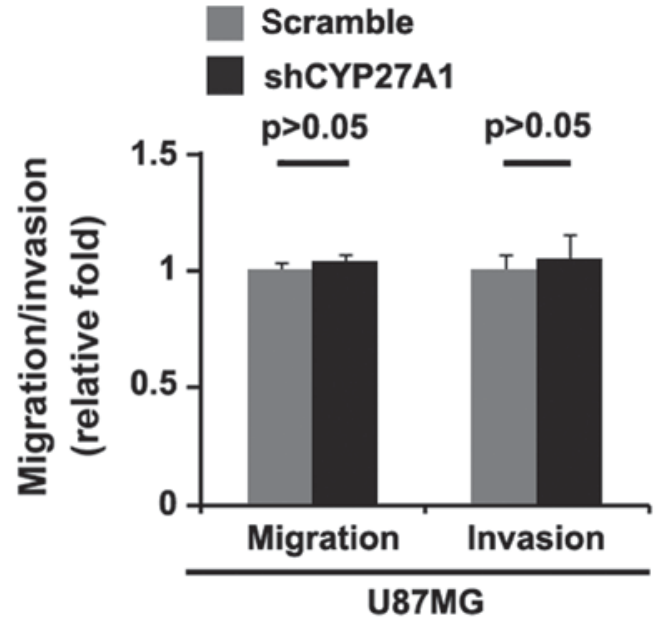

Figure 5. Silencing CYP27A1 does not affect migration and invasion in glioblastoma U87MG cells. Matrigel invasion assay and Transwell migration assay for U87MG cells transfected with shCYP27A1 plasmids orscramble. $\mathrm{n}=3$. Error bars indicate standard error of the mean. CYP27A1, cytochrome P450, family 27 , subfamily A, polypeptide 1 ; sh, short hairpin.

upregulated in glioblastoma and it can promote proliferation in glioblastoma cells, the mechanisms promoting CYP27A1 expression in the disease were then studied. miRNAs are a new class of small ( $22 \mathrm{nt})$ noncoding RNAs, which negatively regulate protein-coding gene expression by targeting mRNA degradation or translation inhibition (15). Downregulation of specific miRNA may contribute to oncogene overexpression (16). Thus, it was reasoned whether CYP27A1 was upregulated by defect of specific miRNA. To confirm this reason, a commonly used prediction algorithm, miRanda (http://www.microrna.org/), was used to analyze the 3 'UTR of CYP27A1.

The algorithm predicted that miR-204 and miR-221 could target the 3'UTR of CYP27A1 and the predicted target is shown in Fig. 7A. To study the biological function of miR-204, it was investigated whether miR-204 expression could be increased by pre-miR-204 in U87MG cells. RT-PCR was performed to detect miR-204 expression in the cells transfected with pre-miR-204 and the results demonstrated that pre-miR-204 could significantly upregulate miR-204 expression in U87MG cells (Fig. 7B). In order to identify that CYP27A1 could be downregulated by miR-204, immunofluorescence and western blot analyses were performed to study whether miR-204 could affect CYP27A1 protein expression. Immunofluorescence analyses demonstrated that CYP27A1 was significantly downregulated in U87MG cells transfected with pre-miR-204 and representative micrographs, and the quantification of immunofluorescence is shown in Fig. 7C. Consistent with immunofluorescence analysis, the results of western blot analysis demonstrated that CYP27A1 protein was significantly downregulated by miR-204 in U87MG cells (Fig. 7D).

In addition, RT-PCR was also performed to detect CYP27A1 mRNA in the cells transfected with pre-miR-204. The results demonstrated that miR-204 downregulated CYP27A1 mRNA level in U87MG cells (Fig. 7E). To demonstrate the direct regulation of CYP27A1 by miR-204, luciferase reporters with the targeting sequences of wild-type (CYP27A1-wt-luc) were used to detect whether miR-204 targets 3'UTR of CYP27A1 mRNA. A luciferase assay was performed. The

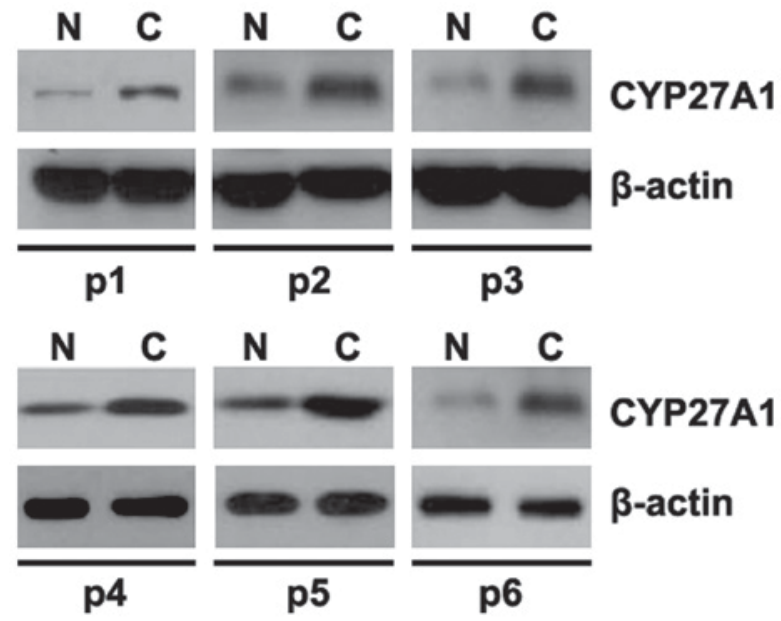

Figure 6. CYP27A1 is upregulated in glioblastoma tissues. Differential expression of CYP27A1 in 6 pairs of human glioblastoma tissues (C) and corresponding adjacent normal tissues $(\mathrm{N})$ were detected by western blot analysis. $\beta$-actin was used as an endogenous control. $n=6$. CYP27A1, cytochrome P450, family 27, subfamily A, polypeptide 1 .

results demonstrated that miR-204 significantly inhibited CYP27A1-WT-luc plasmids in the cells (Fig. 7F). Meanwhile, we observed that miR-204 inhibits CYP27A1 expression in LN-229 glioblastoma cells by targeting 3'UTR of CYP27A1 mRNA (Fig. 8). The results confirmed that miR-204 negatively regulates protein-coding gene CYP27A1 expression by targeting its 3'UTR in glioblastoma cells.

miR-204 is downregulated in glioblastoma and its overexpression inhibits proliferation, migration and invasion in glioblastoma cells. To assess the expression of miR-204 in glioblastoma tissues, northern blot analysis was conducted in 7 pairs of glioblastoma tissues and matched adjacent normal tissue samples. The expression of miR-204 was consistently lower in the glioblastoma tissues compared with normal tissues (Fig. 9A). In an attempt to identify the role of miR-204 in regulating proliferation of U87MG cells, the cells were transfected with pre-miR-204. Following stable transfection, the proliferation rates of U87MG cells were tested by an MTT assay. The results demonstrated that overexpression of miR-204 significantly inhibited the proliferation rate of U87MG cells and that the inhibition of cellular proliferation was dose-dependent (Fig. 9B). This was revealed by BrdU incorporation analysis showing that transfection with miR-204 resulted in decreased DNA synthesis activity per viable cell in U87MG cells (Fig. 9C). Considering that miR-204 evidently inhibited the proliferation of U87MG cells, the present study then sought to determine whether miR-204 would have any impact on migration and invasion in U87MG cells. The migration and invasion assay demonstrated that the overexpression of miR-204 not only inhibited migration of U87MG cells, but also suppressed their invasion (Fig. 9D). Moreover, we observed that miR-204 inhibits proliferation in LN-229 glioblastoma cells (Fig. 10).

\section{Discussion}

Increased CYP27A1 expression was detected in aberrant crypt foci, and adenomatous polyps, as well as the 
A C hsa-miR-204/CYP27A1 Alignment

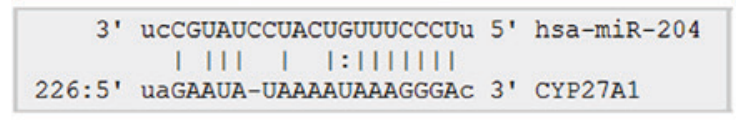

C hsa-miR-211/CYP27A1 Alignment

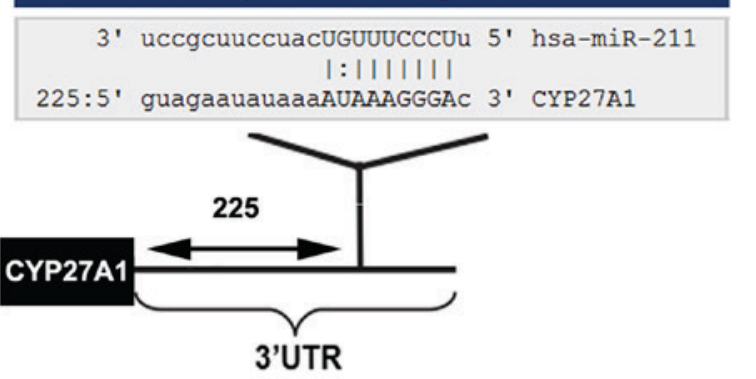

B
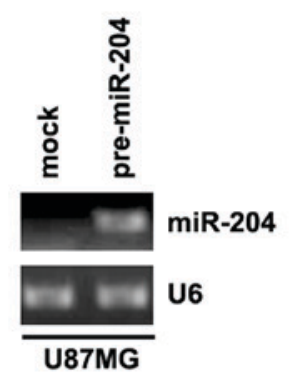

C
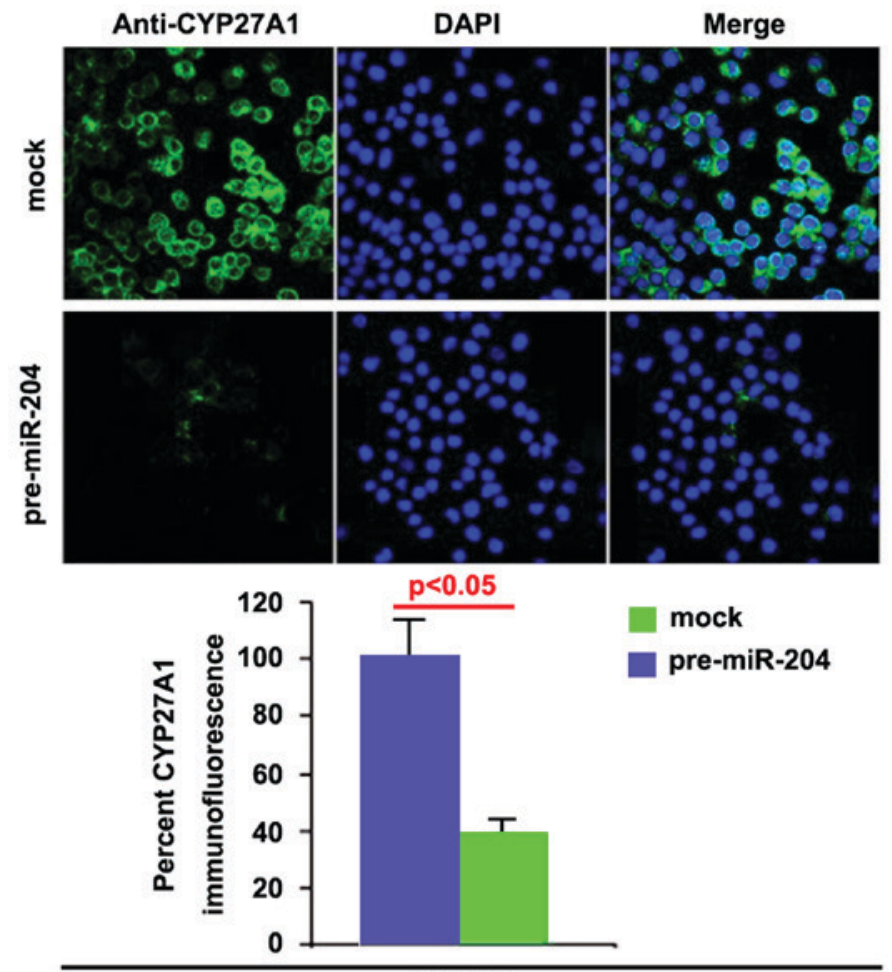

U87MG

D

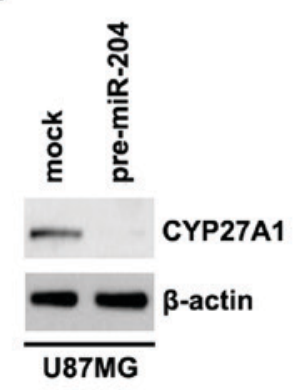

E

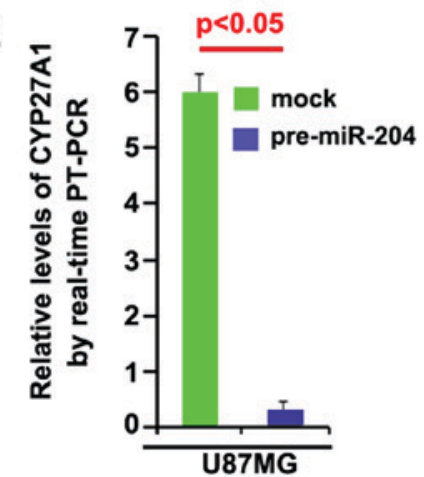

F

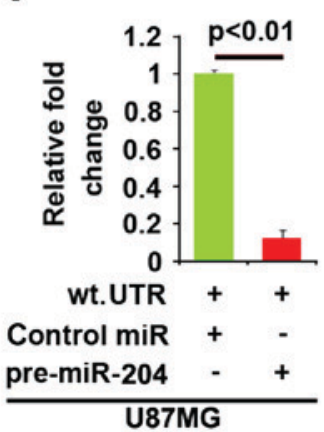

Figure 7. CYP27A1 is a target of miR-204 in glioblastoma U87MG cells. (A) Diagram demonstrating that CYP27A1 is a target gene of miR-204 and miR-211, predicted by miRanda. (B) Detection of miR-204 by RT-PCR in U87MG cells transfected with precursor-miR-204 or the control miR (mock). (C) Immunofluorescence analyses of U87MG cells transfected with pre-miR-204 or the control miR (mock). Upper panel shows microscopic images of immunofluorescence staining of one representative experiment (magnification, x100). Bottom panel shows graphic presentation of mean fluorescence intensities of three independent experiments. (D) Western blot analysis for CYP27A1 in U87MG cells. U87MG cells were transfected with pre-miR-204 or control-miR (mock). $\beta$-actin was used as the loading control. (E) RT-PCR for CYP27A1 in U87MG cells. U87MG cells were transfected with pre-miR-204 or control-miR (mock). GAPDH was a loading control. (F) Reporter assay, with cotransfection of $500 \mathrm{ng}$ WT-reporter and $50 \mathrm{nM}$ control-miR, or pre-miR-204 as indicated $36 \mathrm{~h}$ after transfection, cells were harvested for luciferase reporter assay. + represents existing inserted fragments; - represents no existing inserted fragments; $\mathrm{n}=3$. Error bars indicate standard error of the mean. CYP27A1, cytochrome P450, family 27, subfamily A, polypeptide 1; miR, microRNA; RT-PCR, reverse transcription-polymerase chain reaction; WT, wild-type; has, Homo sapiens; UTR, untranslated region. 
A
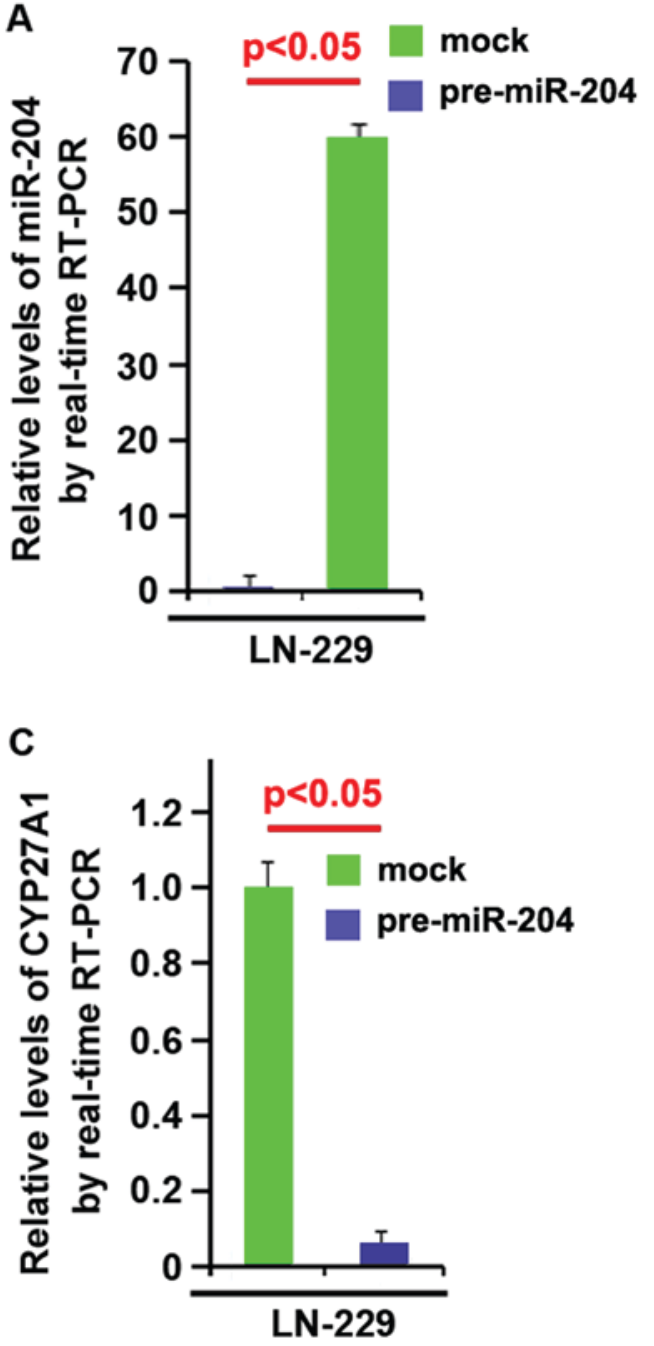

B

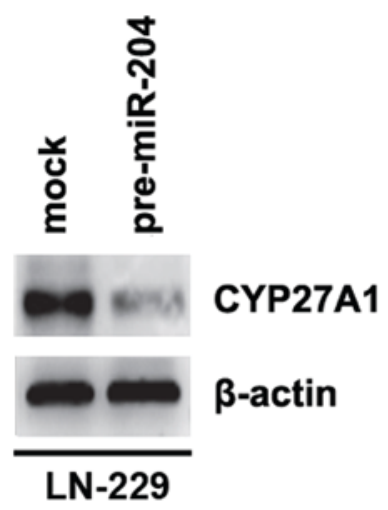

D

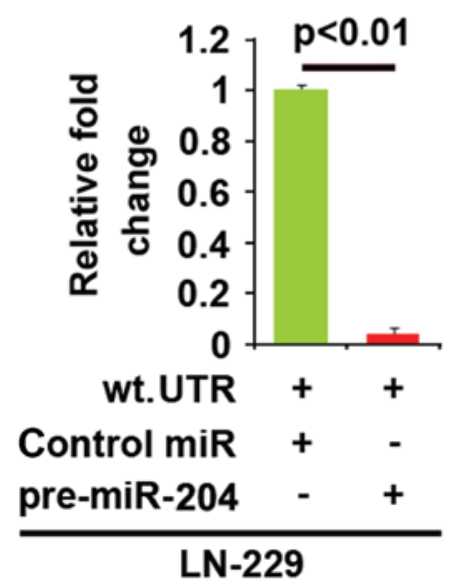

Figure 8. CYP27A1 is a target of miR-204 in glioblastoma LN-229 cells. (A) Detection of miR-204 by RT-PCR in LN-229 cells transfected with pre-miR-204 (precursors miRNA) or the control miR (mock). (B) Western blot analysis for CYP27A1 in LN-229 cells. LN-229 cells were transfected with pre-miR-204 or control-miR (mock). $\beta$-actin was used as the loading control. (C) RT-PCR for CYP27A1 in LN-229 cells. LN-229 cells were transfected with pre-miR-204 or control-miR (mock). GAPDH was a loading control. (D) Reporter assay, with cotransfection of $500 \mathrm{ng}$ WT-reporter and $50 \mathrm{nM}$ control-miR, or pre-miR-204 as indicated. $36 \mathrm{~h}$ after transfection, cells were harvested for luciferase reporter assay. + represents existing inserted fragments; - represents no existing inserted fragments; $n=3$. Error bars indicate standard error of the mean. CYP27A1, cytochrome P450, family 27, subfamily A, polypeptide 1; miR, microRNA; RT-PCR, reverse transcription-polymerase chain reaction; WT, wild-type.

expression of CYP27A1 protein is associated with tumor grade in breast cancer $(4,14)$. In the present study, it was identified that overexpression of CYP27A1 promoted proliferation in glioblastoma cells and silencing it inhibited proliferation, indicating that it may be an oncogene. However, its overexpression and silencing it did not affect migration and invasion in glioblastoma cells. PDCD4, a known tumor suppressor gene, has been identified as a functional target of miR-21 $(15,16)$. Silencing of SOX2 in glioblastoma tumor-initiating cells causes the cessation of proliferation and loss of tumorigenicity (17). It was observed that CYP27A1 overexpression significantly downregulated PDCD4 and upregulated the SOX2 protein in glioblastoma cells, indicating that CYP27A1 may perform an important role in the regulation of tumor-initiating cells.

In addition, CYP27A1 was found to be upregulated in glioblastoma tissues. However, only collected 6 pairs of glioblastoma tissues and adjacent normal tissues were collected. Thus, future studies should use a bigger sample size.
Previous studies have reported that miR-204 is a tumor suppressor miRNA: miR-204 can suppress head and neck tumor metastasis (18); higher tumor grade of human clear cell renal cell carcinomas was associated with a concomitant decrease in miR-204 (19); loss of miR-204 expression enhances glioma migration and stem cell-like phenotype (20); miR-204 targets B-cell lymphoma-2 expression and enhances responsiveness of gastric cancer (21); and miR-204 downregulates sirtuin 1 (SIRT1) and reverts SIRT1-induced epithelial-mesenchymal transition, anoikis resistance and invasion in gastric cancer cells (22). Dysregulation of miR-204 mediates migration and invasion of endometrial cancer by regulating fork head box C1 (23). miR-204 increases sensitivity of neuroblastoma cells to cisplatin and is associated with a favorable clinical outcome (24). Consistent with these previous studies (18-24), it was identified that miR-204 was not only downregulated in glioblastoma, and inhibited proliferation, migration and invasion in glioblastoma cells. The present study also revealed that overexpressing miR-204 inhibited CYP27A1 expression 

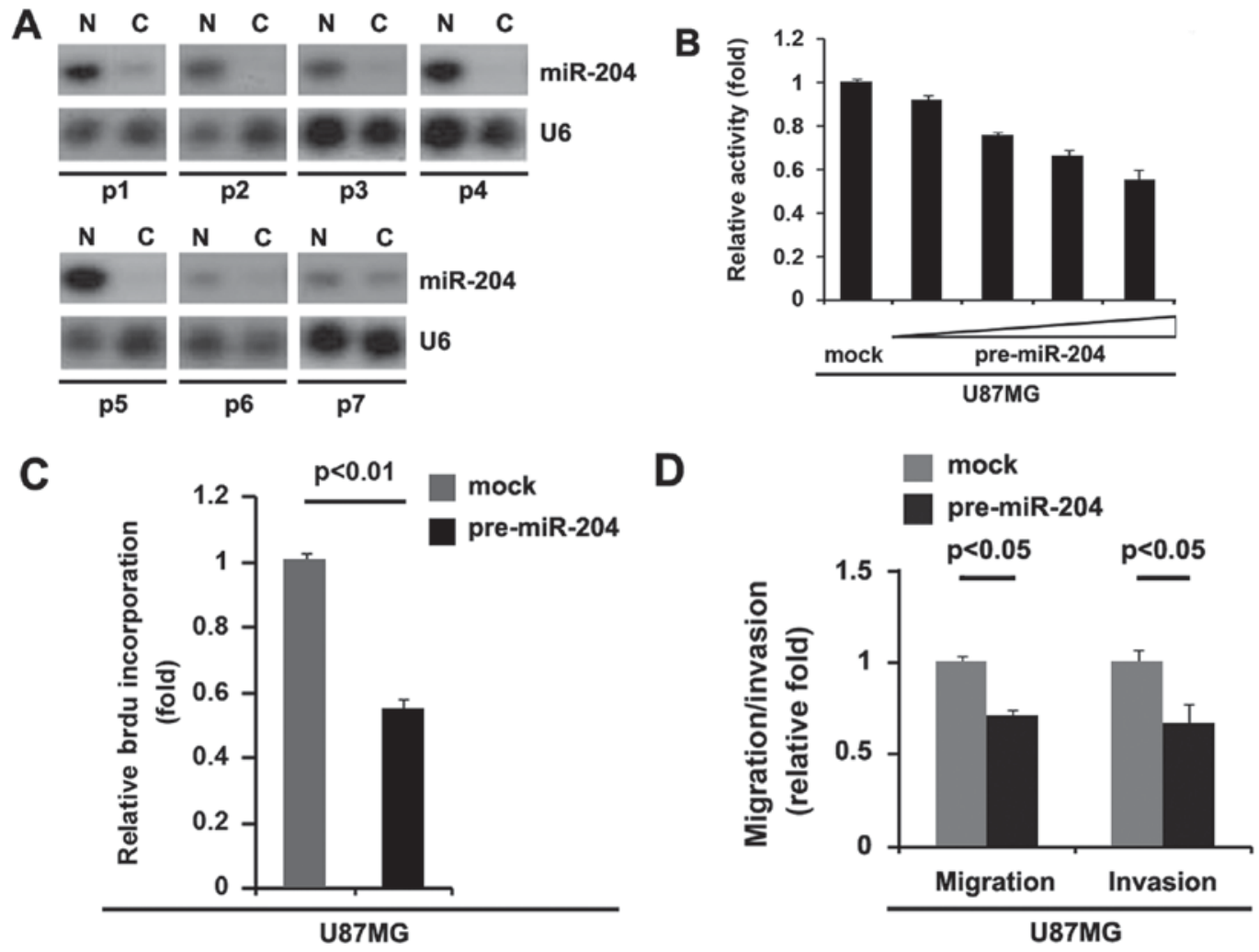

Figure 9. miR-204 is downregulated in glioblastoma and its overexpression inhibits proliferation, migration and invasion in glioblastoma U87MG cells. (A) Northern blot analysis for miR-204 in glioblastoma tissues (C) and adjacent normal tissues (N). $\beta$-actin was used as the loading control. $n=7$. (B) MTT assay for U87MG cells. U87MG cells were transfected with pre-miR-204 (precursors miRNA) and control miR (mock) and cell viability was then measured at the indicated time points using an MTT assay. $\mathrm{n}=3$. (C) BrdU incorporation assay for U87MG cells transfected with pre-miR-204 or control miR (mock). $\mathrm{n}=3$. (D) Matrigel invasion assay and Transwell migration assay for U87MG cells transfected with pre-miR-204 or control miR (mock). $\mathrm{n}=3$. Error bars indicate standard error of the mean. miR, microRNA; BrdU, bromodeoxyuridine.

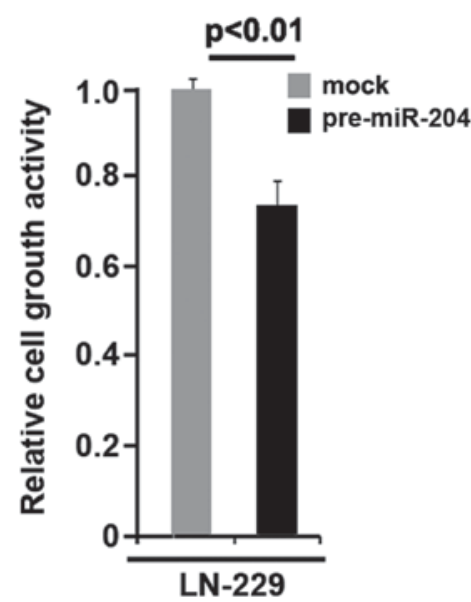

Figure 10. Overexpression of miR-204 inhibits proliferation in glioblastoma LN-229 cells. MTT assay for LN-229 cells. LN-229 cells were transfected with pre-miR-204 and control miR (mock) and cell viability was then measured at the indicated time points using an MTT assay. n=3. Error bars indicate standard error of the mean.

in glioblastoma cells, indicating that the downregulation of miR-204 is associated with the upregulation of CYP27A1 in the disease. It was concluded that miR-204 inhibited proliferation by suppressing CYP27A1 expression. However, it was observed that CYP27A1 did not affect migration and invasion in glioblastoma cells, although miR-204 inhibited migration and invasion. This observation indicated that there are other target genes regulated by $\mathrm{miR}-204$, which may regulate migration and invasion in glioblastoma cells.

Recently, the U-87 MG cell line from ATCC was reported to be contaminated or misidentified (25). It has been proposed as a glioblastoma cell line whose origin is unknown (25). However, the U-87 MG cell line is still widely used for glioblastoma research (25). In the present study, U87MG and LN-229 cells were used. The results were same from the 2 cell lines. Thus, the contamination or misidentification does not affect the conclusion presented.

Elucidating the mechanism by whichmiR-204 inhibits proliferation by suppressing CYP27A1 may help to improve the understanding of the molecular mechanism of proliferation in glioblastoma. Thus, restoration of miR-204 may represent a promising therapeutic way to inhibit CYP27A1-mediated proliferation regulation. However, the roles of CYP27A1 require confirmation in vivo.

\section{Acknowledgements}

Not applicable.

\section{Funding}

The present study was supported by Yishui Central Hospital and Linyi People's Hospital. 


\section{Availability of data and materials}

The datasets used and/or analyzed during the current study are available from the corresponding author on reasonable request.

\section{Authors' contributions}

JX and LQT conceived the study, collected the experimental data and wrote a draft of the manuscript. LMZ, DKS, XFL, and PX contributed to the experimental work and data analysis. All authors edited and approved the final version of the manuscript.

\section{Ethics approval and consent to participate}

Ethics approval was obtained from ethics committee of Yishui Central Hospital. All subjects provided written informed consent at the time of enrollment.

\section{Consent for publication}

Consent for publication was obtained from each patient.

\section{Competing interests}

The authors declare that they have no competing financial interests.

\section{References}

1. Siegel RL, Miller KD and Jemal A: Cancer statistics, 2018. CA Cancer J Clin 68: 7-30, 2018.

2. Das S and Marsden PA: Angiogenesis in glioblastoma. N Engl J Med 369: 1561-1563, 2013.

3. Stupp R, Hegi ME, Mason WP, van den Bent MJ, Taphoorn MJ, Janzer RC, Ludwin SK, Allgeier A, Fisher B, Belanger K, et al: Effects of radiotherapy with concomitant and adjuvant temozolomide versus radiotherapy alone on survival in glioblastoma in a randomised phase III study: 5-year analysis of the EORTC-NCIC trial. Lancet Oncol 10: 459-466, 2009.

4. Nelson ER, Wardell SE, Jasper JS, Park S, Suchindran S, Howe MK, Carver NJ, Pillai RV, Sullivan PM, Sondhi V, et al: 27-Hydroxycholesterol links hypercholesterolemia and breast cancer pathophysiology. Science 342: 1094-1098, 2013.

5. Norlin M, von Bahr S, Björkhem I and Wikvall K: On the substrate specificity of human CYP27A1: Implications for bile acid and cholestanol formation. J Lipid Res 44: 1515-1522, 2003

6. Lu DL, Sookthai D, Le Cornet C, Katzke VA, Johnson TS, Kaaks R and Fortner RT: Reproducibility of serum oxysterols and lanosterol among postmenopausal women: Results from EPIC-Heidelberg. Clin Biochem 52: 117-122, 2018.

7. Björkhem I: Mechanism of degradation of the steroid side chain in the formation of bile acids. J Lipid Res 33: 455-471, 1992.

8. Russell DW and Setchell KD: Bile acid biosynthesis. Biochemistry 31: 4737-4749, 1992.

9. Lee RC, Feinbaum RL and Ambros V: The C. elegans heterochronic gene lin-4 encodes small RNAs with antisense complementarity to lin-14. Cell 75: 843-854, 1993.
10. Chan JA, Krichevsky AM and Kosik KS: MicroRNA-21 is an antiapoptotic factor in human glioblastoma cells. Cancer Res 65: 6029-6033, 2005.

11. Mao J, Zhang M, Zhong M, Zhang Y and Lv K: MicroRNA-204, a direct negative regulator of ezrin gene expression, inhibits glioma cell migration and invasion. Mol Cell Biochem 396: $117-128,2014$.

12. Kefas B, Godlewski J, Comeau L, Li Y, Abounader R, Hawkinson M, Lee J, Fine H, Chiocca EA, Lawler S and Purow B: microRNA-7 inhibits the epidermal growth factor receptor and the Akt pathway and is down-regulated in glioblastoma. Cancer Res 68: 3566-3572, 2008.

13. Livak KJ and Schmittgen TD: Analysis of relative gene expression data using real-time quantitative PCR and the 2(-Delta Delta C(T)) method. Methods 25: 402-408, 2001.

14. Matusiak D and Benya RV: CYP27A1 and CYP24 expression as a function of malignant transformation in the colon. J Histochem Cytochem 55: 1257-1264, 2007.

15. Lu Z, Liu M, Stribinskis V, Klinge CM, Ramos KS, Colburn NH and Li Y: MicroRNA-21 promotes cell transformation by targeting the programmed cell death 4 gene. Oncogene 27: 4373-4379, 2008.

16. Zhu S, Wu H, Wu F, Nie D, Sheng S and Mo YY: MicroRNA-21 targets tumor suppressor genes in invasion and metastasis. Cell Res 18: 350-359, 2008.

17. Gangemi RM, Griffero F, Marubbi D, Perera M, Capra MC, Malatesta P, Ravetti GL, Zona GL, Daga A and Corte G: SOX2 silencing in glioblastoma tumor-initiating cells causes stop of proliferation and loss of tumorigenicity. Stem Cells 27: 40-48, 2009.

18. Lee Y, Yang X, Huang Y, Fan H, Zhang Q, Wu Y, Li J, Hasina R, Cheng C, Lingen MW, et al: Network modeling identifies molecular functions targeted by miR-204 to suppress head and neck tumor metastasis. PLoS Comput Biol 6: e1000730, 2010.

19. Mikhaylova O, Stratton Y, Hall D, Kellner E, Ehmer B, Drew AF, Gallo CA, Plas DR, Biesiada J, Meller J and Czyzyk-Krzeska MF: VHL-regulated MiR-204 suppresses tumor growth through inhibition of LC3B-mediated autophagy in renal clear cell carcinoma. Cancer Cell 21: 532-546, 2012.

20. Ying Z, Li Y, Wu J,Zhu X, Yang Y, Tian H, Li W, Hu B, Cheng SY and Li M: Loss of miR-204 expression enhances glioma migration and stem cell-like phenotype. Cancer Res 73: 990-999, 2013.

21. Sacconi A, Biagioni F, Canu V, Mori F, Di Benedetto A, Lorenzon L, Ercolani C, Di Agostino S, Cambria AM, Germoni S, et al: miR-204 targets Bcl-2 expression and enhances responsiveness of gastric cancer. Cell Death Dis 3: e423, 2012.

22. Zhang L, Wang X and Chen P: miR-204 down regulates SIRT1 and reverts SIRT1-induced epithelial-mesenchymal transition, anoikis resistance and invasion in gastric cancer cells. BMC Cancer 13: 290, 2013.

23. Chung TK, Lau TS, Cheung TH, Yim SF, Lo KW, Siu NS, Chan LK, Yu MY, Kwong J, Doran G, et al: Dysregulation of microRNA-204 mediates migration and invasion of endometrial cancer by regulating FOXC1. Int J Cancer 130: 1036-1045, 2012.

24. Ryan J, Tivnan A, Fay J, Bryan K, Meehan M, Creevey L, Lynch J, Bray IM, O'Meara A, Tracey L, et al: MicroRNA-204 increases sensitivity of neuroblastoma cells to cisplatin and is associated with a favourable clinical outcome. Br J Cancer 107: 967-976, 2012.

25. Allen M, Bjerke M, Edlund $\mathrm{H}$, Nelander S and Westermark B: Origin of the U87MG glioma cell line: Good news and bad news. Sci Transl Med 8: 354re3, 2016.

This work is licensed under a Creative Commons Attribution-NonCommercial-NoDerivatives 4.0 International (CC BY-NC-ND 4.0) License. 